Bull. Mater. Sci., Vol. 17, No. 4, August 1994, pp. 395-398. (C Printed in India.

\title{
Critical analysis of driving force for pure-step migration by vacancy supersaturation and crystal anisotropy
}

\author{
V HARI KRISHNA \\ Department of Metallurgical Engineering, K.R.E.C., Surathkal 574 157, India
}

\begin{abstract}
The gist of the paper is to introduce the other compelling factors for the boundary displacement which are dominant in the lower temperature regime such as non equilibrium vacancy concentration and anisotropy and to prove that the driving force due to the above factors is sufficient to induce grain boundary migration.
\end{abstract}

Keywords. Vacancy supersaturation; junction dipole; anisotropy; osmotic pressure; stress tensor.

In the systematic study of diffusion-induced grain migration the boundary migration by the formation of ledges is not uncommon (Glieter and Calmer 1972; King 1991). The high migration rate in diffusion-induced recrystallization and diffusion-induced grain migration is in turn associated with the formation of pure steps (Babcock and Balluffi 1981, 1989). A pure step can be viewed as a microfacet with junction dislocation dipole. In the presence of vacancy supersaturation the net force exerted on the junction dipole is equal to the net difference of the climbing force across the dipole and is given by (King 1981)

$$
F / L=\frac{k T b_{e}}{\Omega} \ln \left\{C_{v} /\left[C_{v}+\mathrm{d} C_{v} / \mathrm{d} x\right]\right\}
$$

where $b_{e}$ is the edge component of the burgers vector, $C_{v}$ the vacancy concentration of the step and $\Omega$ the atomic volume. As can be seen from the above equation, the magnitude of the force depends on the large vacancy supersaturation which is temperature-dependent and hence cannot be dominating in the low-temperature regime (King 1981).

We take in the present study two additional compelling factors for the pure-step displacement: (a) nonequilibrium vacancy concentration at the boundary and (b) crystal anisotropy. The osmotic force due to nonequilibrium vacancy at the boundaries is given by Hirth and Lothe (1986)

$$
F^{o s} / L=\frac{k T b_{e}}{\Omega} \ln \left(C_{v} / C_{v o}\right)
$$

where $C_{v o}$ is the equilibrium vacancy concentration. At equilibrium vacancy concentration the climb forces across the junction dipole are equal in value and opposite in direction and hence a net force, but this force couple produces a stressed state in the vicinity of the riser plane as shown in figure 1 . The uniaxial stress $\sigma_{x x}$ in the dislocation slip plane produces an elastic force per unit dislocation length (Hirth and Lothe 1986)

$$
F^{e l}=\sigma_{x x} b_{e},
$$




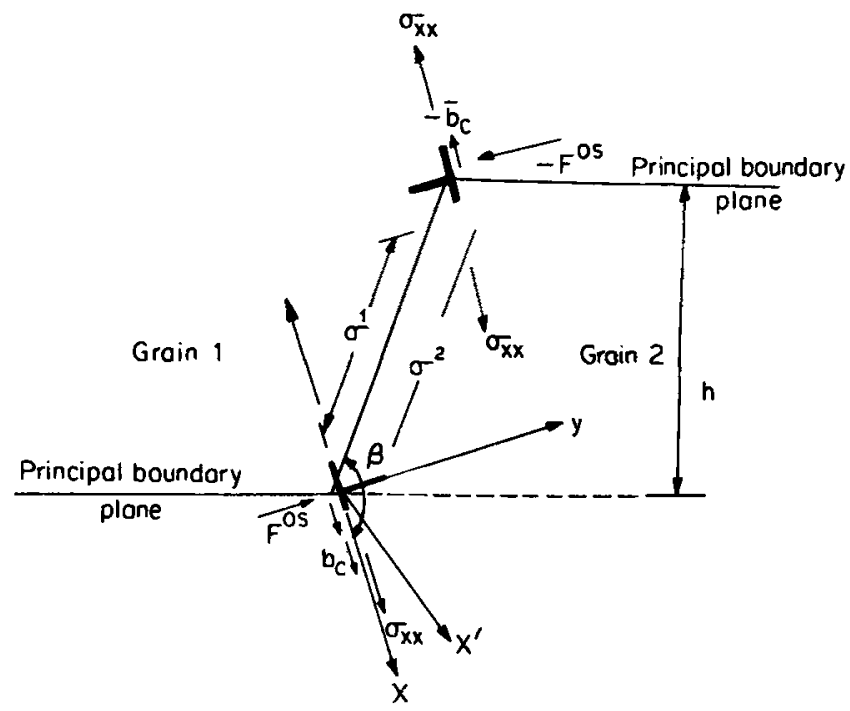

Figare 1. Distribution of force and stress at the function dipole across the slip plane.

which counteracts the osmotic force (2). At equilibrium $F^{o s}=F^{e l}$, so (2) and (3) give the stress $\sigma_{x x}$ as

$$
\sigma_{x x}=\frac{k T}{\Omega} \ln \left(C_{v} / C_{v o}\right)
$$

For $C_{v}>C_{p o}$ the stresses are tensile and for $C_{v}<C_{v 0}$ compressive. The tensile force increases the chemical potential of the vacancies at the junctions, thus mobilizing them at the boundary far from dislocations (Hirth and Lothe 1986)

$$
\mu=\sigma_{x x} \Omega=k T \ln \left(C_{v} / C_{v o}\right),
$$

which leads to zero flux by conservation and relaxes the step structure. Since adjacent grains represent a pair of misoriented crystals, the applied stress $\sigma_{i j}$ would cause in the presence of anisotropy unequal strains in regions of the opposite sides of the riser plane. The misfit strains may be written as

$$
\begin{aligned}
e_{i} & =n_{j}\left(\varepsilon_{i j}^{1}-\varepsilon_{i j}^{2}\right) \\
& =n_{j}\left(S_{i j k l}^{1}-S_{i j k l}^{2}\right) \sigma_{k l},
\end{aligned}
$$

where $n_{j}$ 's are the components of the normal to the riser, $\varepsilon_{i j}$ is the strain tensor and $S_{i j k l}$ the compliance tensor in the grain $m(m=1,2)$.

The additional hydrostatic stress due to the anisotropy assuming equilibrium for cubic symmetry is

$$
\sigma_{H}^{1}=-\sigma_{H}^{2}=\sigma_{x x} \theta_{A}^{1} / 3 S_{12}^{1} * \frac{\sin ^{2} \phi \cos ^{2} \phi \cos 2 \beta}{1+\theta_{A}^{1} S_{12}^{1} \sin ^{2} \phi \cos ^{2} \phi},
$$


$\theta_{A}^{1}=S_{11}^{1}-S_{12}^{1}-2 S_{44}^{1}$ is the parameter of anisotropy in grain (1), $\phi$ the grain misorientation. In the case of $\beta<3 \pi / 4$ and $\theta_{A}^{1}<0$ the stress $\sigma_{H}^{1}$ will be tensile and compressive in the other grain.

Nonequilibrium vacancies are absorbed by the step and their energy is converted into the elastic energy of the stressed step. So, the chemical potential gradient across the grain boundary can be due to the elastic anisotropy as considered above and due to the nonequilibrium vacancy concentration. These gradients in chemical potential do work for the step movement in the process.

Thus in the above cases the additional stresses induce a gradient in chemical potential of the atoms across the grain boundary,

$$
\begin{aligned}
\nabla \mu & =\Omega \Delta \sigma_{H} \\
& =2 \Omega \sigma_{H} / \delta,
\end{aligned}
$$

where $\delta$ is the boundary width. The work done for each atom crossing the boundary corresponds to the free-energy change

$$
\begin{aligned}
\Delta G_{1-2}^{v} & =\Omega \Delta \sigma_{H} \\
& =2 \Omega \sigma_{H} .
\end{aligned}
$$

The step movement is in the opposite direction to the flow of atoms which are from the compressive to tensile zone. Comparing the nonequilibrium vacancy concentration $\Delta G_{1-2}^{v}$ (King 1981) and anisotropy $\Delta G_{1-2}^{A}$ we have

$$
\begin{aligned}
\frac{\Delta G_{1-2}^{v}}{\Delta G_{1-2}^{A}} & =K\left\{\ln \left[C_{v} /\left(C_{v}+h \mathrm{~d} C_{v} / \mathrm{d} x\right)\right]\right\} /\left[\ln C_{v} / C_{v o}\right], \\
K & =\frac{3 S_{1-2}^{1} b_{e}\left[1+\sin ^{2} \phi \cos ^{2} \phi * \theta_{A}^{1} / S_{1-2}^{1}\right]}{2 \theta_{A}^{1} * h *\left[\sin ^{2} \phi \cos ^{2} \phi \cos 2 \beta\right]}
\end{aligned}
$$

In interdiffusion experiments $\Delta C_{v}-C_{v v} \ll C_{v o}$. Hence (8) can be approximated to

$$
\frac{\Delta G_{1-2}^{v}}{\Delta G_{1-2}^{A}}=\frac{K C_{v o} h \mathrm{~d} C_{v} / \mathrm{d} x}{\Delta C_{v} C_{v}}=K \mathrm{~d} / h,
$$

where $\mathrm{d}$ is the distance over which the vacancy concentration changes from $C_{v}$ to $C_{v o}$.

Let us now calculate the VA driving force to the grain boundary motion (King 1989)

$$
\Delta G^{\text {curr }} / \Omega=2 \Gamma / R
$$

associated with the grain boundary curvature where $\Gamma$ is the grain boundary free energy and $1 / R=1 / R_{1}+1 / R_{2}$ where $R_{1}$ and $R_{2}$ are the radii in two mutually perpendicular directions. Assuming

$$
\begin{aligned}
& \Gamma=0.5 \mathrm{~J} / \mathrm{m}^{2}, \\
& R=10^{-7} \mathrm{~m}, \\
& \Omega=1.5 * 10^{-29} \mathrm{~m}^{3},
\end{aligned}
$$

we have $G^{\mathrm{curr}} 1.5 * 10^{-22} \mathrm{~J}$, under vacancy supersaturation $C_{v} / C_{v o}=0.01$ at $T=500$ 
and for the above values of the parameters,

$$
\Delta G_{1-2}^{v}=\left(3 K b_{e} / 2 \mathrm{~h}\right) k T * \ln \left(C_{v} / C_{v o}\right)=2 \cdot 5 * 10^{-22} \mathrm{~J}
$$

Thus the VA driving force can induce the grain boundary migration away from the centre of curvature even for grains as small as $0.1 \mu \mathrm{m}$. So we conclude that VA driving force plays a significant role in the movement of pure steps in grain boundaries.

\section{References}

Gleiter H and Calmer B 1972 High angle boundaries (Oxford: Pergamon Press) p. 119

King A H 1991 Metal Trans. 221177

Babcock S E and Balluffi R W 1989 Acta Metall. 372357

Babcock S E and Balluffi R W 1981 Acta Metall. 372367

King A H 1981 Scr. Metall. 151221

King A H 1989 Int. Mater. Rev. 32173

Hirth J P and Lothe J 1986 Theory of dislocations (New York)

McLean D 1957 Grain boundaries in materials (Oxford: Clarendon Press) p. 346 